\title{
The Planetary Nebulae Populations in the Local Group
}

\author{
Magda Arnaboldi
}

\begin{abstract}
Planetary nebulae have been used as tracers of light and kinematics for the stellar populations in early-type galaxies since more than twenty years. Several empirical properties have surfaced: for example the invariant bright cut-off of the planetary nebulae luminosity function and correlations of the luminosity specific PN number with the integrated properties of the parent stellar populations. These observed properties are poorly understood in terms of a simple model of a ionized nebula expanding around an non-evolving central star. In order to make further steps, we need to study self-contained systems at know distances whose PN populations are sufficiently nearby to permit investigation into their physical properties. The galaxies in the Local Group represent a valid proxies to study these late phases of evolved stellar populations with a spread of metallicities, $\alpha$-element enhancements, and star forming histories.
\end{abstract}

\section{Introduction}

Galactic planetary nebulae (PNs) are roughly $0.3 \mathrm{pc}$ in diameter. Hence when they are observed in external galaxies at distances larger than $50 \mathrm{Kpc}$ they are identifiable as unresolved emissions of monochromatic green light at $5007 \AA$, that is the strongest optical emission line of the [OIII] doublet. About 2000 PNs are known in the Milky Way (MW) out of 200 billion stars and are found mostly in the plane of the MW. In our own galaxy, 95\% of the stars end their lives as PNs, and the remaining 5\% as SNs. Single stars can be observed in their PNs phase because the ionized envelope of a $\mathrm{PN}$ is able to re-emit up to $15 \%$ of the core star UV radiation in one single optical line, the [OIII] 5007 $\AA$ line, which can be detected in narrow band imaging surveys against the continuum light emitted by the rest of the stars. The total integrated flux in the [OIII] 5007 line emitted by a nebula can be expressed

Magda Arnaboldi

European Southern Observatory, e-mail: marnaboleeso.org 
as

$$
m_{5007}=-2.5 \log \left(F_{5007}\right)-13.74
$$

When these magnitudes are measured for a PN population, they provide the planetary nebulae luminosity functions (PNLF). In external galaxies, the PNLFs show several empirical properties, for example the bright cut-off is observed to be empirically invariant and the overall shape seems to be reproduced by the Ciardullo's 1989 analytical formula

$$
N(M) \propto \exp (0.307 M) \times(1-\exp (3(M *-M))) ; M *=-4.51
$$

\subsection{The PN.S Key Project on Early-Type Galaxies}

PNs have been used as tracers of light and motions in the outer regions of earlytype galaxies since more than 20 years, starting from the early models of the mass distribution in M32 by Nolthenius \& Ford (1986) to the current Planetary Nebulae Spectrograph (PN.S) - Key Project - for the survey of PNs in earlytype galaxies (ETGs). Such survey includes all ETGs within $20 \mathrm{Mpc}$, at airmass less than 1.5 which are observable from the William Herschel telescope on La Palma, Spain. This survey includes 20 ETGs, from E0 to E4, 11 S0s and it is now expanded to include spiral galaxies. Observational data are presented in Coccato et al. (2009) and Cortesi et al. (2013); data products can be downloaded at http://www.strw.leidenuniv.nl/pns/PNS_public_web/PN.S_data.html

The current results of these studies illustrate that the PN number density follows the surface brightness of the stars that dominate the light in these galaxies, and whenever PNs and absorption line kinematics overlap, they agree in all sample galaxies surveyed thus far. The results from the kinematical studies show that the specific angular momentum in the outer halo, out to $10 R_{e}$, where $R_{e}$ is the effective radius of the surface brightness distribution, correlates with the properties of the high surface brightness regions, but there are significant deviations (Coccato et al. 2009, Cortesi et al. 2013). Furthermore, the velocity r.m.s. dispersion profiles for the galaxies in this sample show a dichotomy between flat and quasi Keplerian dispersion profiles, see Figure 1. The question whether there is a continuum or a separation between the two families is still open, and is going to be addressed by the new data release from the PN.S key project. Contrary to the initial claims of quasi Keplerian dispersion profile signaling lower dark matter content in these systems ( e.g. Romanowsky et al. 2003), these galaxies can arbor a dark matter halo consistent with $\Lambda C D M$ and their steep $\sigma$ profiles at large radii are the results of the $\mathrm{R}^{1 / 4}$ surface brightness profile and strong radial anisotropy in the outer parts (de Lorenzi et al. 2009, Morganti et al. 2013). 


\subsection{PNs in ETGs}

The luminosity specific PN number, or $\alpha$ parameter for short is given by

$$
\alpha=N_{P N} / L_{\odot, B o l}
$$

and it is a characteristic number that associates the PN population with its parent stellar population emitting the bulk of the light in an extragalactic system. In the simple stellar population theory, such number is also given by

$$
\alpha=N_{P N} / L_{\odot, B o l}=B \tau_{P N}
$$

whether $B$ is the specific evolutionary flux and $\tau_{P N}$ is the PN visibility lifetime of a PN. In the simple model of a uniformly expanding nebula around a non-evolving core (Henize \& Westerlund 1963), $\tau_{P N}=D / v_{\text {exp }}$, where $D$ is the diameter of a PN when its $m_{5007}$ has faded 8 mags below the bright cut-off, and $v_{\exp }$ is the nebula expansion velocity. For this simple model $\tau_{P N}=30000 \mathrm{yrs}$. Empirically, the $\alpha$ parameter shows a constant value in stellar populations with integrated $B-V<0.8$, and then the scatter increases for stellar populations with redder colors (Hui et al. 1993, Ciardullo et al. 2002, Buzzoni et al. 2006, Cortesi et al. 2013). Specific to ETGs, the $\alpha$ parameter correlates with $F U V-V$ colors, so that fewer PNs are observed in systems with an $F U V-V$ color excess, see Figure 2 ,

Fig. 1 Comparison between radial profiles of $V_{r m s}$ (top panel) and velocity dispersion (bottom panel) of the sample galaxies, obtained by combining the stellar and PNs kinematics along the major axis. Profiles have been scaled to the effective radius and normalized to their value at $1.0 R_{e}$. Colors are chosen in order to highlight the contrast between lines and thus better distinguish different profiles. The velocity r.m.s. and $\sigma / \sigma_{0}$ profiles in ETGs as traced by PNs. From the PN.S Key Project, Coccato et al. (2009).

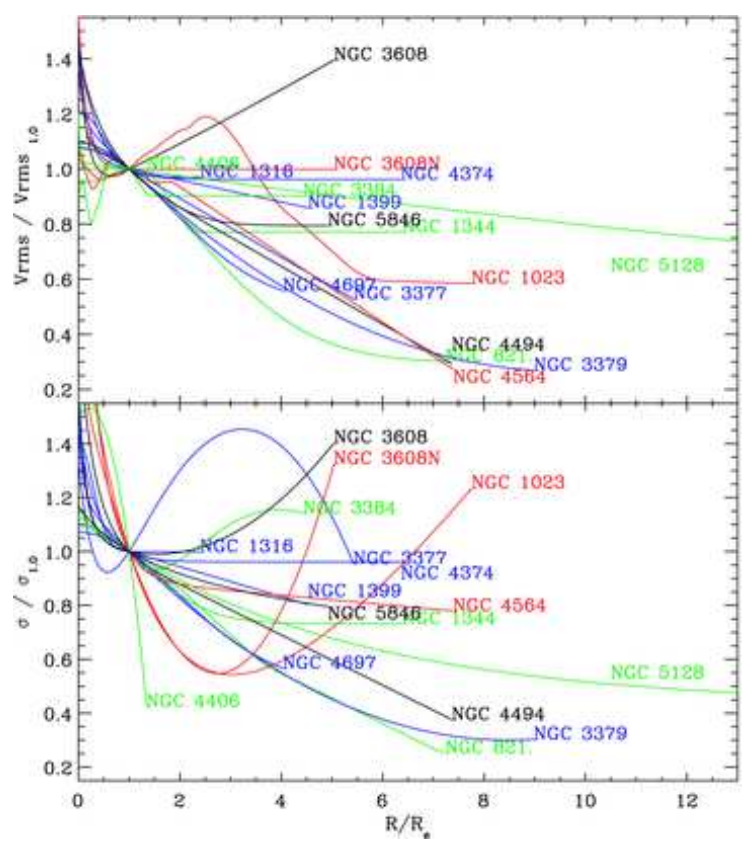


From the relation between $\alpha$ and $\tau_{P N}$, a lower value of $\alpha$ translates into a shorter visibility lifetime for the PN population of about $1000-5000 \mathrm{yrs}$ in those systems where the low values of $\alpha$ are measured.

\section{Surveys of PNs in the Local Group}

In this section, a summary overview of the available surveys for PNs in the Local Group (LG) is provided, with emphasis on the surveys of the MW PNs, in the Large Magellanic Cloud (LMC) and the Andromeda galaxy (M31).

Survey of PNs in the MW - The PN candidates in the MW are selected in $\mathrm{H} \alpha$ and confirmed spectroscopically. The most recent survey is the Macquarie/AAO/Strasbourg $\mathrm{H} \alpha$ Planetary Nebula Catalog (MASH I+ MASH II) with $\sim 1200$ PNs detected (Frew et al. 2013, Parker et al. 2006 (MASH I), and Miszalski et al. 2008 (MASH II)). These latest results build on and expand the Strasbourg/ESO catalog of PN ( 2000 PNs by Acker et al. 1992). The PN population that is specific to the Bulge includes 802 PNs, with 405 PNs from MASH I + II and 396 PNs from Acker et al. (1992). Independent surveys of the Bulge PNs (373 PNs) were carried out by Beaulieu et al. (1999). Weidmann et al. (2013) also identified PNs in the MW bulge from VVV survey data (about 381 PNs).

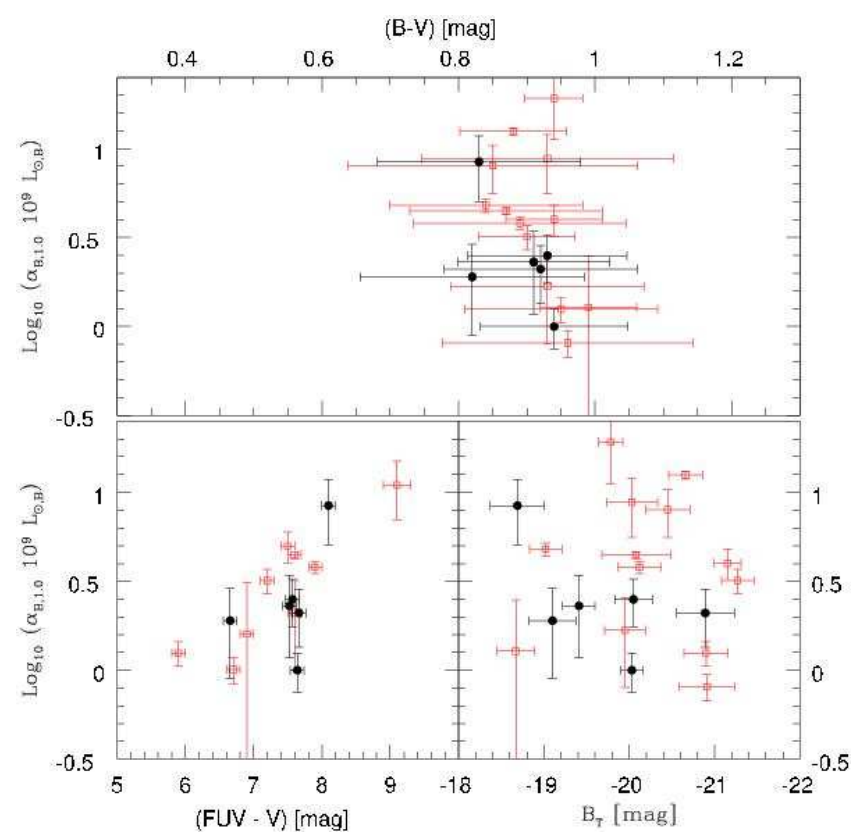

Fig. 2 The $\alpha$ parameter values measured in ETGs and correlations with $B-V$ color, total B magnitude and $F U V-V$ color. From Cortesi et al. (2013). 
Survey of PNs in the Large Magellanic Cloud - An extended survey in the LMC was carried out by Reid \& Parker (2010) in the central $25 \mathrm{deg}^{2}$ area. A total of 740 PNs were detected and spectroscopically confirmed with the AAT 2dF Spectrograph. The magnitude range covered by the sample spans 10 mags down the bright cut-off of the PNLF.

Survey of PNs in M31 - The extensive survey of PNs in M31 was carried out with the Planetary Nebulae Spectrograph (PN.S) by Merrett et al. (2006), with the discovery of $2730 \mathrm{PN}$ candidates. For this sample, the PNLF is complete 4 mag down the brightest cut-off (Merrett et al. 2006).

Survey of PNs in LG galaxies - The PN populations in LG galaxies were surveyed and detected in $16 \mathrm{LG}$ members (Reid 2012). There are no PN detected in LG galaxies with $M_{V}<-9.8\left(\simeq 10^{6} L_{\odot}\right)$. Empirically, it is consistent with a maximum specific PN luminosity number of $\alpha_{\max }<1 P N / 10^{7} L_{\odot, B o l}$.

\section{Physical properties of the PN populations - visibility lifetimes and planetary nebulae luminosity functions}

MW Bulge - The distribution of angular diameter in arcsec for Bulge and Disk PNs is available from the MASH-I + II survey, with $90 \%$ of the whole population within the maximum angular diameter of $35 \mathrm{arcsec}$. In the case of the PN sample restricted to the MW Bulge, the plot $\log (S B)$ vs. $\log (D)$ shows that round PNs are the brightest and most compact objects, with average diameter of $0.3 \mathrm{pc}$ : clearly these PNs are those preferentially detected when PN populations are observed in external galaxies.

There are $560 \mathrm{PNs}$ in the Galactic Bulge with average diameter about $0.3 \mathrm{pc}$. The PN visibility lifetime $\tau_{P N}$ for this volume limited sample can be estimated using $v_{\text {exp }}$ and $D_{P N}$, as $D_{P N} / v_{\text {exp }}$. Given that the average expansion velocity of Bulge PNs is $30 \mathrm{kms}^{-1}$, thus the observable lifetime of a Galactic Bulge PN is then only a few $10^{3}$ years, which is consistent with visibility lifetime of PN samples in ETGs.

Kovacevic et al. (2011) carried out the [OIII] narrow band imaging of the PN population in the central $10^{\circ} \times 10^{\circ}$ region towards the MW Bulge. This sample accounts for $80 \%$ of known PNs in this region. Fluxes and diameters are measured from narrow band imaging with the MOSAIC-II camera on the 4-m Blanco Telescope at the CTIO. They surveyed $60 \mathrm{deg}^{2}$ uniformly with a narrow band filter centered on the [O III] $\lambda 5007$ line; 104 objects were used for calibration of the $m_{5007}$ magnitudes of 228 PNs. At $8 \mathrm{kpc}, m *$ is $10.00(M *=-4.51)$, and one can ask whether the PN population associated with an old $\alpha$-element enhanced parent stellar population follows the PNLF analytical formula suggested by Ciardullo et al. (1989). The empirical PNLF for the MW Bulge is steeper than what is described by the Ciardullo's 1989 analytical formula, in a way similar to what is observed in the halo of M87 (Longobardi et al. 2013). For more detail, we refer to Arnaboldi, Longobardi, Gerhard in prep.

LMC - Reid \& Parker (2010) build the PNLF for the extended sample of PNs in the LMC. In order to use the PNLF for distance determination, they corrected 
the [OIII] fluxes from the confirmed spectra for line-of-sight reddening only. While the bright cut-off is consistent with $\mathrm{M}^{*}=-4.51$, once corrected for the metallicity dependence, the Ciardullo's 1989 formula is a poor fit to the empirical PNLF. The major departure from the analytical formula occurs 1.5 mags from the bright cut-off and lasts for $1.5 \mathrm{mag}$. The empirical PNLF is flatter in these magnitude ranges than what predicted by the double exponential formula (eq. 2). This very sensitive survey illustrates another new feature of the PNLF: a peak in the PN number density which is caused by a rapid rise 4 mags below the brightest PN. This feature is characteristics of models where the [OIII] $5007 \AA$ emission is driven by the rapid evolution of the central star. The peak and the steep descent represents the point at which the central star starts its trajectory down towards the white dwarf cooling track. Such a peak in the PNLF is predicted by the models of Meńdez et al. (1993)

The very deep survey of PNs in the LMC shows that Ciardullo's analytical formula fails to reproduce the faint end of the PNLF, with candidates being detected up to 2 mags fainter than the expected limit of 8 mag down the brightest PNs.

M31 - The PN population in M31 illustrates the strength of PNs as tracers of light and motions of the stars that dominate the bulk of the light emitted in this galaxy. As shown by Merrett et al. (2006), the PN number density profile follows the surface brightness profile along the major and the minor axis out to 2 and 1 degree, respectively, from the galaxy center. The PNLF for the entire population is complete 4 magnitudes down the bright cut-off and it is well fitted by eq. 2. Deviations from the Ciardullo's analytical formula are measured for the PNLF in the very central bright region by Pastorello et al. (2013). The kinematics of the PN sample shows the presence of a substructure (Merrett et al. 2003), while the bulk of the PN follow the stellar disk, with a clear signature for the asymmetric drift and a flattening of $\sigma_{v}$ at large radii in the disk, which is signaling an increase in the DM content.

$L G$ galaxies - The surveys of PN population in the LG galaxies can provide useful information on the frequency of PNs in different systems, and whether there are systems with higher specific frequencies as function of luminosities and morphological types. These analysis were carried out by Reid (2012) and Coccato et al. (2013); they show that galaxy systems with luminosities less than $10^{8} \mathrm{~L}_{\odot, B o l}$ have fewer PNs that the number predicted from the theoretical maximum luminosity specific PN number of $1 P N / 1.5 \times 10^{6} \mathrm{~L}_{\odot}$ (Buzzoni et al. 2006). The observed number of PNs approaches the theoretical predictions for LMC-like and $\mathrm{L}^{*}$ systems, see Figure 3 .

\section{Physical properties of the PN populations in old-metal rich vs. poor-star forming stellar populations.}

The oldest and most metal rich stellar populations are found in the bright, red, and passively evolving galaxies at the centers of massive clusters. The nearest such object is the galaxy M87, in the core of the Virgo cluster. Narrow band imaging surveys by Jacoby et al. (1990) provided the first PN candidates in M87, and the first PNs as- 
sociated with the intracluster light (ICL) were discovered by Arnaboldi et al. (1996) and Feldmeier et al. (1998). The spectroscopic confirmation based on the detection of the [OIII] 4959/5007 $\AA$ doublet in single objects was obtained by Arnaboldi et al. (2004) and Doherty et al. (2009). These 52 PNs turned out to be associated to the halo of M87 and ICL, as indicated by their line-of-sight velocities.

An important step forward in the study of PNs in M87 and the Virgo cluster comes with the availability of SuprimeCAM on the Subaru telescope, that allows the complete coverage of $0.25 \mathrm{deg}^{2} \mathrm{FoV}$, with the collecting power of an 8 meter telescope. The results for the PN populations in M87 and ICL are reported in Longobardi et al. (2013) and Arnaboldi et al. (2003).

The deep narrow band imaging survey carried out by Longobardi et al. (2013) led to the identification of $688 \mathrm{PN}$ candidates in the outer regions of M87, covering a radial range from 10 to $150 \mathrm{kpc}$. This sample is corrected for the contamination by Ly $\alpha$ emitters at redshift 3.14, [OII] emitters at redshift 0.34 , faint continuum stars, and for color and spatial incompleteness. Longobardi et al. (2013) built the PN number density profile and compared it with the surface brightness profile of M87 in the V band. Deviations were clearly detected with the number density profile being flatter than the surface brightness profile at large radii. Such behavior was reproduced by a two component model where the PNs in the survey field come from the PN population associated with the M87 halo and the ICL. A two component model reproduces the observed profile when $\alpha_{2.5, I C L}=3 \times \alpha_{2.5, M 87}$. The values of $\alpha$ translate into PN visibility lifetimes that are $\tau_{P N}=1.4 \times 10^{4}$ for PNs in the ICL and $4.5 \times 10^{3}$ for PNs in the M87 halo.

Longobardi et al. (2013) used their large PN sample in the outer regions of M87 to investigate the properties of the PNLF. They compared it with the PNLF predicted from Ciardullo's 1989 analytical formula and a distance modulus of 30.8. They reported strong deviations at 1.5 mag below the brightest cut-off with the ob-

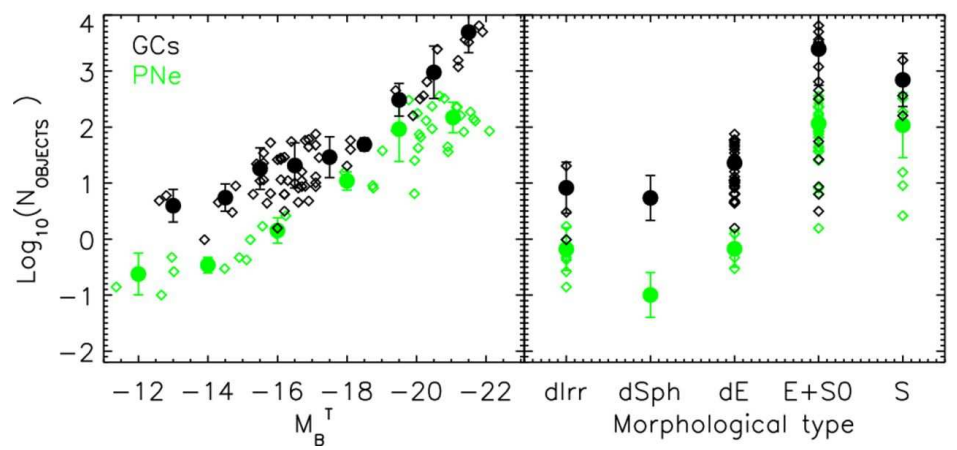

Fig. 3 Expected number of sources (GCs or PNs) as a function of galaxy absolute magnitude (left) and morphology (right). The expected number of GCs and PNs is computed from: (i) the specific frequencies of GCs and PNs and (ii) the total galaxies luminosities in the V and B bands, for GCs and PNs, respectively. The observed number of PNs approaches the theoretical predictions from single-stellar-population models (Buzzoni et al. 2006) for LMC-like and L* systems. From Coccato et al. (2013). 
served gradient being steeper than what is predicted by the analytical formula in this magnitude range. Longobardi et al. (2013) compared the PNLFs extracted in three radial bins, and showed that the three PNLFs have high Kolmogorov-Smirnov (KS) probabilities of being drawn from the same distribution, while the KS test rejects the Ciardullo's formula, see Figure 4

The results are significant for old stellar populations because the steepening of the PNLF is thus shown to be present in all three radial bins, while it is known that the ICL contributes mostly at the largest radius. We can then compare the observed properties of the PNLF in M87 with the PNLF in the M31 bulge, which was used to calibrate the analytical formula from Ciardullo et al. (1989). The PN sample for M31 and the halo of M87 are normalized by the sampled luminosity and then corrected for the distance modulus. In Figure 5, the results show that within one mag of the bright cutoff, the M87 population has fewer PNs than the PNLF of M31, i.e. the slope of the PNLF for the halo of M87 is steeper towards fainter magnitudes.

Fig. 4 Top panel: empirical PNLFs in three radial ranges corrected for color and detection incompleteness: PN candidates within $6.5^{\prime}$ from M87 center triangles, PN candidates between $6.5^{\prime}$ and $13.5^{\prime}$ from M87 center diamonds, PN candidates in the outermost region (distances greater than $13.5^{\prime}$ ) squares. The respective Ly $\alpha$ contribution expected in each radial bin was subtracted. Magnitudes are binned in $0.3 \mathrm{mag}$ bins and the error bars represent the $1 \sigma$ uncertainty from counting statistics combined with the uncertainty from cosmic variance in the Ly density. The red solid line is the convolved analytical formula of Ciardullo et al. (1989) for distance modulus 30.8. Lower panel: same as for the upper plot, but now the three PNLFs are normalized at the total number of objects in each radial bin. The three data sets are consistent with being drawn from the same underlying distribution. From Longobardi et al. (2013).
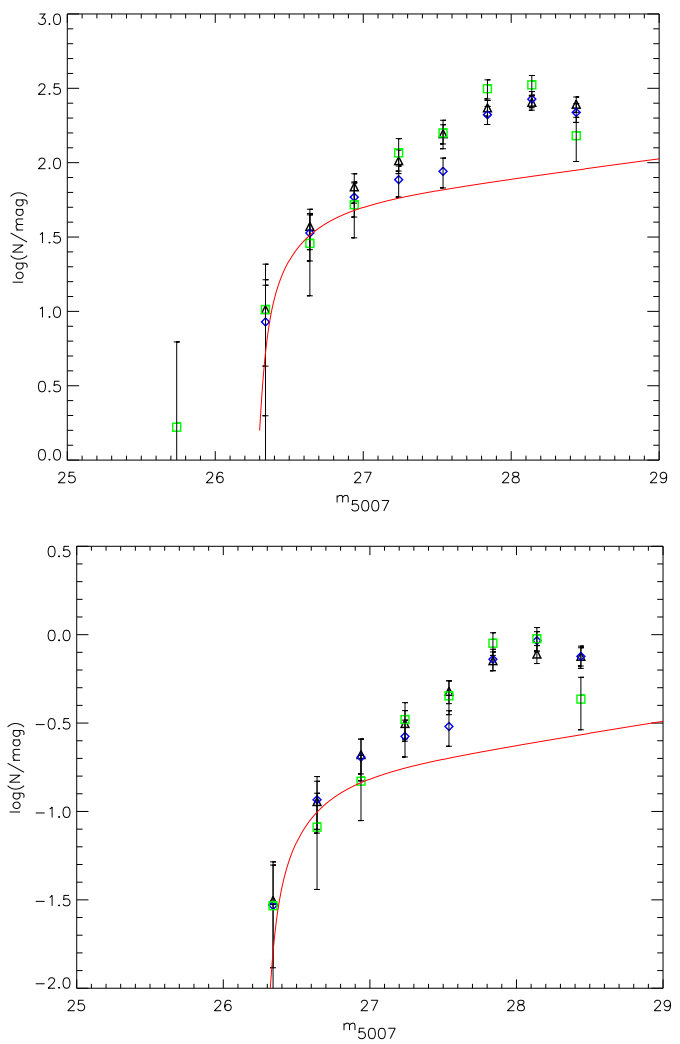
In old stellar populations, one expects the PN central stars to be mostly low mass cores, with $M_{\text {core }} \leq 0.55 \mathrm{M}_{\odot}$. Thus, the slope of the PNLF may turned out steeper than the slope predicted by the fading of a uniformly expanding sphere ionized by a non-evolving star (Henize \& Westerlund 1963). The comparison between the PNLFs of M87 and M31 may indicate that the M87 halo hosts a stellar population with a larger fraction of low mass cores, with respect to the M31 bulge. Comparison of the properties of PNLFs and their gradients $1.5 \mathrm{mag}$ below the brightest PNs is further explored in Arnaboldi, Longobardi, Gerhard in prep.

\section{Conclusions}

The recent work on the PN populations in the halo of M87 and ICL shows that a second parameter is needed to generalize the Ciardullo's 1989 formula and account for population effects. In addition to the distance modulus and the normalization coefficient $c_{1}$, we need to vary the slope $c_{2}$, which is related to the gradient of the PNLF at magnitudes fainter then the PNLF cut-off.

Furthermore, one concludes 1) that PN populations are ubiquitous - deep photometry and kinematics from PNs show that they trace the bulk of star light in LG galaxies, early-type galaxies and in extended luminous halos, 2) that PN populations and their PNLFs show dependencies on stellar populations which are quantified by the gradient in the PNLF at magnitudes below the bright cut-off, and 3) that $\mathrm{M}^{*}$ is empirically observed to be invariant in the systems studied thus far.

Acknowledgements MAR would like to thank her collaborators for their enthusiasm and support, in particular Alessia Longobardi, Ortwin Gerhard and Lodo Coccato. MAR would like to thank the Scientific Organizing Committee for the invitation to give this review and the opportunity to take part in this very special and vibrant conference. MAR acknowledges ESO for financial support. All the best to Bruce and David!

Fig. 5 Luminositynormalized PNLFs for the bulge of M31 (blue triangles) and the halo of M87 (black circles). Data are binned into 0.25 mag intervals. The red line shows the improved fit with the second parameter $c_{2}$, in addition to the distance modulus and the normalization factor $c_{1}$. The M87 PNLF has a steeper slope towards faint magnitudes than M31. From Longobardi et al. (2013).

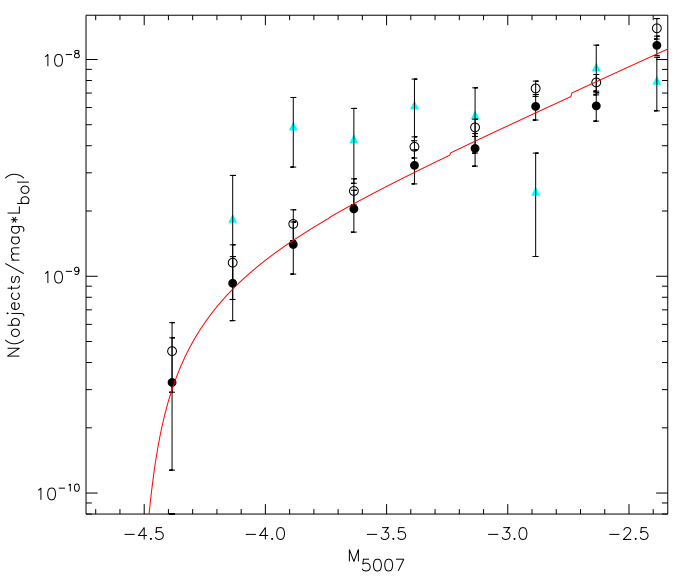




\section{References}

Acker, A., Marcout, J., Ochenbein, F. et al. 1992, Strabourg-ESO Catalogue of Galactic Planetary Nebulae (Garching:ESO)

Arnaboldi, M., Freeman, K., Mendez, R., et al. 1996, ApJ, 472, 175

Arnaboldi, M., Freeman, K., Okamura, S., et al. 2003, AJ, 125, 514

Arnaboldi, M., Gerhard, O., Aguerri, J. , et al. 2004, ApJL, 614, 33

Beaulieu, S., Dopita, M., Freeman, K. 1999, ApJ, 515, 610

Buzzoni, A., Arnaboldi, M., Corradi, R. 2006, MNRAS, 368, 887

Ciardullo, R., Jacoby, G., Ford, H. et al. 1989, ApJ, 339, 53

Ciardullo, R., Feldmeier, J., Jacoby, G. 2002, ApJ, 577, 31

Coccato, L., Arnaboldi, M., Gerhard, O. 2013, MNRAS, 436, 1322

Coccato, L., Gerhard, O., Arnaboldi, M. et al. 2009, MNRAS, 394, 1249

Cortesi, A., Arnaboldi, M., Coccato, L. et al. 2013, A\&A, 549, 115

Doherty, M., Arnaboldi, M., Das, P., et al. 2009, A\&A, 502, 771

de Lorenzi, F. Gerhard, O., Coccato, L., et al. 2009, MNRAS, 395, 76

Feldmeier, J., Ciardullo, R., Jacoby, G. 1998, ApJ, 503, 109

Frew, D., Bojiĉić, I., Parker, Q. 2013, MNRAS, 431, 2

Henize, K. \& Westerlund, B. 1963, ApJ, 1963, 137, 747

Hui, X. Ford, H., Ciardullo, R., et al. 1993, ApJ, 414, 463

Longobardi, A., Arnaboldi, M., Gerhard, O., et al. 2013, A\&A, 558, 42

Jacoby, G., Ciardullo, R., Ford, H. 1990, ApJ, 356, 332

Kovacevic, A., Parker, Q., Jacoby, G., et al. 2011, MNRAS, 414, 860

Mendez, R.H., Kudritzki, R., Ciardullo, R., et al. 1993, A\&A, 275, 534

Merrett, H., Kuijken, K., Merrifield, M., et al. 2003, MNRAS, 346, 62

Merrett, H., Merrifield, M., Douglas, N., et al. 2006, MNRAS, 369, 120

Miszalski, B., Parker, Q., Acker, A., et al. 2008, MNRAS, 384, 525 (MASH II)

Morganti, L., Gerhard, O., Coccato, L., et al. 2013, MNRAS, 431, 357

Nolthenius, R. \& Ford, H. 1986, ApJ, 305, 600

Parker, Q., Acker, A., Frew, D., et al. 2006, MNRAS, 373, 79 (MASH I)

Pastorello, N., Sarzi, M., Cappellari, M., et al. 2013, MNRAS, 430, 121

Reid, W. 2012, IAU Symp., 283, 227

Reid, W \& Parker, Q. 2010, MNRAS, 405, 1349

Romanowsky, A., Douglas, N. Arnaboldi, M., et al. 2003, Science, 301, 1696

Weidmann, W., Gamen, R., van Hoof, P., et al. 2013, A\&A, 552, 74 